\title{
Disenfranchised grief and Covid-19: How do we make it less painful?
}

\author{
SMITHA RAMADAS, SHARANYA VIJAYAKUMAR
}

\begin{abstract}
The devastating effects of death due to Covid-19 on the bereaved are not adequately addressed. The grief associated with death during the Covid-19 pandemic is disenfranchised and complicated and has significant repercussions on the bereaved. The lockdown, social distancing norms, isolation due to disease or quarantine and infectivity of the disease, place restrictions on the traditional mourning practices. Misconceptions also play a role. Dignity and ethics are frequently breached, perhaps inadvertently. This can lead to serious mental and physical health consequences. We explore the complexities and suggest measures for acknowledging the grief and making it less painful. Pragmatic suggestions to avoid emotional distancing and to uphold the dignity and rights of the deceased and the bereaved are highlighted with examples which can be emulated.
\end{abstract}

Keywords: Covid-19, disenfranchised grief, pandemic deaths, bereavement, rights of the deceased

\section{Disenfranchised grief and Covid-19}

As of November 1, 2020, the WHO estimates that there are $1,125,245$ confirmed cases of Covid-19, including 528,204 deaths globally. The ensuing grief of the survivors is not adequately explored or addressed. When the loss is unexpected and sudden (1), it can have a significant negative impact on the immediate and long-term quality of life of close family members. This can trigger the onset or relapse of psychiatric and physical disorders (2). This pandemic has the potential to lead to a second mental health pandemic.

Grief associated with Covid-19 is often disenfranchised. Disenfranchised grief is "the grief that persons experience when they incur a loss that is not or cannot be openly acknowledged, publicly mourned, or socially supported" (3). Here the proper expression of grief is curtailed. The impact of the loss on the person's life goes unaddressed and the grieving process is inhibited. The grief in the Covid-19 scenario occurs against the backdrop of multiple losses like loss of normal life, jobs, security and physical intimacy.

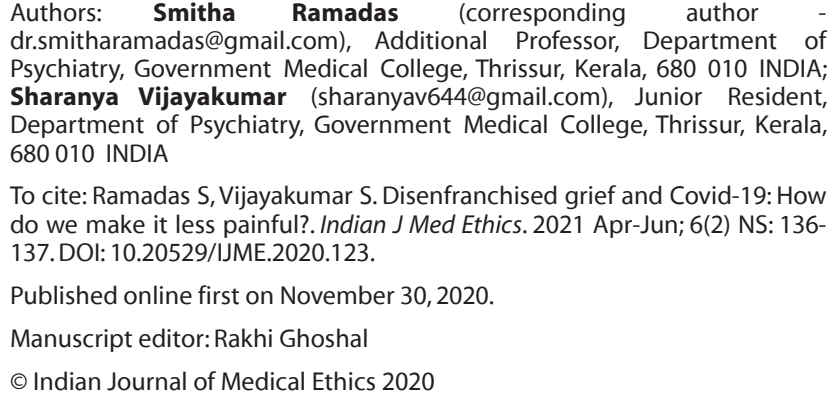

To cite: Ramadas S, Vijayakumar S. Disenfranchised grief and Covid-19: How do we make it less painful?. Indian J Med Ethics. 2021 Apr-Jun; 6(2) NS: 136137. DOI: 10.20529/IJME.2020.123.

Published online first on November 30, 2020.

Manuscript editor: Rakhi Ghoshal

(c) Indian Journal of Medical Ethics 2020

The protocol of Covid-19 cremation limits the number of people who can attend the funeral. The lockdown, with its attendant social and travel restrictions prevents people from physically reaching out to the bereaved or reaching on time to bid goodbye to the deceased. The absence of traditional bereavement practices and rituals has its negative effects on the grieving process.

The misconceptions surrounding the spread of infection from a dead body are another matter of concern. The humiliating experiences surrounding the burial of a doctor who died of Covid-19 infection in Chennai were deeply disturbing. The protest and physical violence against the relatives and colleagues who carried the body highlights the misconceptions among people (4). The loss of dignity of the deceased and bereaved can have manifold repercussions. Usually, the close family of the deceased would be quarantined, which deprives them of social support and disrupts the mourning process. The ensuing loneliness also inhibits mourning. Loneliness has also been highlighted as a risk factor and predictor of detrimental mental and physical health consequences of bereavement (5).

The healthcare providers who are already exhausted with work overload, and living with the constant fear of contracting infection themselves, may have compromised capacity to express sensitivity and empathy to the bereaved relatives. They may also be affected by secondary and vicarious trauma (6) which again jeopardises caregiving.

Fear of contracting the disease along with grief due to loss of their loved one, would contribute to the emergence of mental health problems in the bereaved. Isolation during the pandemic can have adverse effects on the quality of life of the person. If isolated away from home, their normal routines of life are lost, apart from the fear of economic loss and displacement. Further, if they test positive for the disease that would mean extended physical isolation, inability to share grief with close family or friends and perhaps the imminent threat of death. In the hospital setting, witnessing deaths nearby can also precipitate anguish. Such situations cause traumatic disruption of social connectedness and intimacy, which are very essential to recovery.

The grief of the survivors of people who die overseas, with no family members nearby is a complex one. The mortal remains of the dead may never be seen by their kith and kin, making the grief deeply traumatic and disenfranchised. Grief inhibited can prolong misery and lead to adverse mental and physical problems

\section{How do we make it less painful?}

Social distancing, should not lead to emotional isolation of the bereaved. The need of the hour is a multidimensional 
healthcare approach. Efficient support systems are to be established, catering to the emotional, material, economic and other psychosocial needs of the survivors. Tele counselling services for the bereaved need to be made accessible to them. Grief helplines similar to suicide helplines should be put in place.

Providing timely and accurate information to the family of the patient, about his/her deteriorating health status empathetically would prepare the family for better acceptance of the inevitable. Handling the dead body should be done with sensitivity and dignity.

\section{Recommendations}

We offer some suggestions for more empathetic handling of bereavement in Covid-19 times:

- While preparing the dead body for the last journey, the decedent's fresh set of personal clothing may also be loosely draped over the decontaminated plastic body bags, to give a personalised feel and dignity to the dead. The face end may be unzipped at the burial/cremation ground, to allow the relatives to have a last look (7).

- The bereaved may be allowed sufficient time to say goodbye, adhering to the Covid-19 safety protocols. An incident in Kerala in which the body of a woman who died due to non Covid-19 illness was kept in the mortuary, for her daughter to complete her quarantine and fondly pay her last respects is a striking example of preserving the dignity and rights of the bereaved (8). This can also provide closure to the bereaved, which helps in the grieving process.

- Facilities for live streaming of the funeral can be provided. Allowing religious rituals according to the cultural background and needs of the family, incorporating safety protocols should be facilitated. The family may be permitted to collect the ash and pursue the last rites (7). Such measures which approximate the traditional death rites and rituals may bestow a sense of normalcy to the bereaved relatives and friends.

- Restricted visits and virtual family meeting may be encouraged. Social connectedness needs to be fostered maintaining physical distancing and safety precautions. The lockdown should not lead to emotional "breakdown" of the bereaved. If a large number of deaths results in a shortage of space for burial, bodies may be allowed to be cremated in a socially and religiously sanctioned manner. The religious authorities need to adopt a flexible attitude. In Thrissur district of Kerala, the arch bishop permitted cremation of dead bodies of Covid-19 patients due to space constraints for deep burial, permitting the ash to be brought to the cemetery (9). Such practices need to be emulated.

\section{Conclusions}

It is essential to spread awareness among the public, to clear misconceptions and prevent stigmatisation of patients, families, and the dead. All frontline staff should be trained to provide culturally sensitive emotional support for a dignified exit to the dying and to treat the bereaved sensitively (10). The principle of humanistic healthcare "To cure sometimes, treat often and comfort always" needs to be adopted. Mental healthcare should be integrated with the usual care to provide holistic healthcare. The support of mental health professionals and counsellors needs to be enlisted and continued whenever needed.

Such measures help to preserve the dignity of the deceased and the bereaved and provide a sense of closure to the bereaved. This may help them accept the loss, process the grief and move on in life.

\section{References}

1. Lundin T. Morbidity following sudden and unexpected bereavement. Br J Psychiatry. 1984 Jan; 144: 84-8.

2. Shear MK. Getting straight about grief. Depress Anxiety. 2012 Jun;29(6): 461-4

3. Doka KJ.Disenfranchised grief. In: Doka KJ, Editor. Disenfranchised grief: Recognizing hidden sorrow. Lexington, MA: Lexington Books; 1989. p. 3-11

4. Josephine SM. In Chennai, doctor's burial marred by protests, attacks. The Hindu.com. 2020 Apr 20 [cited 2020 Oct 31]. Available from https://www.thehindu.com/news/cities/chennai/in-chennai-doctorsburial-marred-by-protests-attacks/article31386195.ece

5. Fried El, Bockting C, Arjadi R, Borsboom D, Amshoff M, Cramer AOJ, et al. From loss to loneliness: The relationship between bereavement and depressive symptoms. J Abnorm Psychol. 2015 May; 124(2):25665.

6. Selman LE, Chao D, Sowden R, Marshall S, Chamberlain C, Koffman J. Bereavement support on the frontline of COVID-19: Recommendations for hospital clinicians. J Pain Symptom Manage. 2020 May 4:S0885-3924(20)30244-X. doi: 10.1016/j.jpainsymman. 2020.04.024.

7. Ministry of Health and Family Welfare, Govt of India. COVID-19: Guidelines on Dead Body Management. New Delhi: MoHFW; 2020 Mar 15[cited 2020 Oct 31]. Available from https://www.mohfw.gov.in/ pdf/

1584423700568_COVID19GuidelinesonDeadbodymanagement.pdf

8. Innu quarantine theerum; amaykku anthyachumbanamekan makal [Today quarantine will be over; daughter to give last kiss to mother]. Malayala Manorama, Thrissur. 2020 Jun 20.p. 2. [Malayalam].

9. Special Correspondent. Thrissur Archdiocese allows cremation of COVID victims as a special case. The Hindu.com. 2020 Jun 16[cited 2020 Oct 31]. Available from https://www.thehindu.com/news/ national/kerala/thrissur-archdiocese-allows-cremation-of-covidvictims-as-a-special-case/article31845314.ece

10. Leong IY, Lee AO, Ng TW, Lee LB, Koh NY, Yap E, et al. The challenge of providing holistic care in a viral epidemic: Opportunities for palliative care. Palliat Med. 2004 Jan 18;18(1):12-18. 